\title{
The Temporal Requirements of Directly Observing Self-gravitating Spiral Waves in Protoplanetary Disks with ALMA
}

\author{
Cassandra Hall $^{1,8}$ (D), Ruobing Dong ${ }^{2}$ (D), Ken Rice ${ }^{3,4}$, Tim J. Harries ${ }^{5}$ (D), Joan Najita ${ }^{6}$, Richard Alexander ${ }^{1}$, and Sean Brittain ${ }^{7}$ (D) \\ ${ }^{1}$ Dept. of Physics \& Astronomy, University of Leicester, University Road, Leicester, LE1 7RH, UK; cassandra.hall@le.ac.uk \\ ${ }^{2}$ Department of Physics \& Astronomy, University of Victoria, Victoria BC V8P 1A1, Canada \\ ${ }^{3}$ SUPA, Institute for Astronomy, Royal Observatory Edinburgh, University of Edinburgh, Blackford Hill, Edinburgh, EH9 3HJ, UK \\ ${ }^{4}$ Centre for Exoplanet Science, University of Edinburgh, Edinburgh, UK \\ ${ }^{5}$ Department of Physics and Astronomy, University of Exeter, Stocker Road, Exeter EX4 4QL, UK \\ ${ }^{6}$ National Optical Astronomy Observatory, 950 N. Cherry Avenue, Tucson, AZ 85719, USA \\ ${ }^{7}$ Department of Physics \& Astronomy, 118 Kinard Laboratory, Clemson University, Clemson, SC 29634-0978, USA \\ Received 2018 October 3; revised 2018 December 19; accepted 2018 December 20; published 2019 February 4
}

\begin{abstract}
We investigate how the detectability of signatures of self-gravity in a protoplanetary disk depends on its temporal evolution. We run a one-dimensional model for secular timescales to follow the disk mass as a function of time. We then combine this with three-dimensional global hydrodynamics simulations that employ a hybrid radiative transfer method to approximate realistic heating and cooling. We simulate ALMA continuum observations of these systems and find that structures induced by the gravitational instability (GI) are readily detectable when $q=M_{\text {disk }} / M_{*} \gtrsim 0.25$ and $R_{\text {outer }} \lesssim 100$ au. The high accretion rate generated by gravito-turbulence in such a massive disk drains its mass to below the detection threshold in $\sim 10^{4}$ years, or approximately $1 \%$ of the typical disk lifetime. Therefore, disks with spiral arms detected in ALMA dust observations, if generated by self-gravity, must either be still receiving infall to maintain a high $q$ value, or have just emerged from their natal envelope. Detection of substructure in systems with lower $q$ is possible, but would require a specialist integration with the most extended configuration over several days. This disfavors the possibility of GI-caused spiral structure in systems with $q<0.25$ being detected in relatively short integration times, such as those found in the DSHARP ALMA survey. We find no temporal dependence of detectability on dynamical timescales.
\end{abstract}

Key words: accretion, accretion disks - protoplanetary disks

\section{Introduction}

In the last decade, huge leaps in imaging capabilities have allowed astronomers to obtain high-resolution images of protoplanetary disks, the birth sites of exoplanets. Among them, nearinfrared (NIR) imaging allows us to probe the surface of disks, while dust continuum observations at $\sim \mathrm{mm}$ wavelengths carried out using the Atacama Large Millimeter/submillimeter array (ALMA) probe down to the midplane of disks and trace the density structures in $\sim \mathrm{mm}$-sized dust. Surprisingly, a significant fraction of these disks have substructures, such as rings (e.g., ALMA Partnership et al. 2015) and spirals (e.g., Pérez et al. 2016), and these substructures seem to be common; images of disks from surveys that are spatially resolved down to 28 au show substructure in about $20 \%$ of these objects (Cieza et al. 2019).

It is widely thought that rings may be caused by planetary-mass companions (Kley \& Nelson 2012; Baruteau et al. 2014; Dipierro et al. 2015b, 2018; Dong et al. 2015c; Dong \& Fung 2017). However, at present, we lack the data to distinguish between their formation through planet-disk interactions and other possible mechanisms, such as self-induced dust pileups (Gonzalez et al. 2015) or aggregate sintering (Okuzumi et al. 2016). The origin of spiral features in protoplanetary disks is just as murky (Dong et al. 2018b). Although planets can induce spiral features in scattered light images (Dong et al. 2015b), it is unclear if they can do so in $\sim$ mm emission that trace the distribution of $\sim$ mm-sized dust.

A possible explanation for spirals present in $\sim \mathrm{mm}$ emission is the gravitational instability (GI). At the moment of formation of a star-disk system, the masses of the star and the disk are

\footnotetext{
${ }^{8}$ Author to whom any correspondence should be addressed.
}

comparable, guaranteeing that the system is self-gravitating (Lin \& Pringle 1987, 1990). This ensures that the Toomre parameter, $Q$, of such a system (Toomre 1964)

$$
Q=\frac{c_{\mathrm{s}} \kappa}{\pi G \Sigma} \sim 1
$$

where $c_{\mathrm{S}}$ is the sound speed, $\kappa$ is the epicyclic frequency ( $\kappa=\Omega=\sqrt{G M / r^{3}}$ in a Keplerian disk) and $\Sigma$ is the surface density. So long as $Q \sim 1.5-1.7$, numerical simulations have shown that non-axisymmetric perturbations will grow into spiral waves (Durisen et al. 2007). If the disk is able to cool rapidly relative to the dynamical timescale, the spiral arms may then fragment (Gammie 2001; Rice et al. 2005; Stamatellos et al. 2007; Kratter et al. 2010; Nayakshin 2010; Forgan \& Rice 2013b; Hall et al. 2017; Humphries \& Nayakshin 2018; Forgan et al. 2018a; Stamatellos \& Inutsuka 2018).

Equation (1) is, however, a local condition for instability. Since observations usually give us global properties of a system, it is useful to think about the global requirement for instability, which is simply that the disk-to-star mass ratio

$$
q \equiv \frac{M_{\mathrm{disk}}}{M_{*}}=f \cdot \frac{H}{R} \gtrsim 0.1
$$

(Kratter \& Lodato 2016), where $M_{\mathrm{d}}$ and $M_{*}$ are the mass of the disk and the star, respectively, $H=c_{\mathrm{s}} / \Omega$ is the disk scale height, and $f$ is a numerical prefactor of order unity.

Understanding GI, and subsequent fragmentation, requires observations of protoplanetary disks that are likely to be gravitationally unstable. It has been suggested that some 
systems with spiral arms are gravitationally unstable. For example, the grand design, $m=2$ spiral modes imaged in scattered light in MWC 758 (Benisty et al. 2015) and SAO 206462 (Stolker et al. 2016) are consistent with spirals in GI models (Dong et al. 2015a). On the other hand, the difference between the $q \gtrsim 0.1$ required for the disk to be self-gravitating, and the $q \sim 0.01$ estimated from $\sim \mathrm{mm}$ dust emission (Andrews et al. 2011) leaves this scenario unfavored. However, if part of the disk is optically thick at $\sim \mathrm{mm}$ wavelengths, disk mass could be significantly underestimated (Hartmann et al. 2006; Forgan \& Rice 2013a; Dunham et al. 2014; Evans et al. 2017; Galván-Madrid et al. 2018).

ALMA has recently revealed spiral arms in $\sim \mathrm{mm}$ emission in many systems (Pérez et al. 2016; Tobin et al. 2016; Dong et al. 2018a). The first of its kind, the Elias 2-27 system, is a class II object with an unusually high mm emission-based disk mass estimate- $q \sim 0.24$ (Andrews et al. 2009). It has a twoarmed, grand-design spiral extending out to $R \sim 300$ au from the central star (Pérez et al. 2016). Both GI and an external perturber have been put forward to explain the origin of the spirals (Meru et al. 2017; Tomida et al. 2017), and efforts to distinguish the two are ongoing (Forgan et al. 2018b).

Some previous investigations into spirals detected in ALMA continuum observations have suggested that we should be cautious about assuming they are due to GI. Even if a disk has $q>0.1$, its GI-induced structures are not necessarily detectable, since their amplitudes may not be large enough (Hall et al. 2016). Such features may also be smeared so that their apparent morphology is different to their actual morphology. For example, spiral arms may be smeared into $\sim 2$ when 8-10 are actually present (Dipierro et al. 2014, 2015a). Similarly, although GI models could explain the morphology of the Elias 2-27 system (Meru et al. 2017), fine tuning of the parameter space is needed (Hall et al. 2018), as the extended nature of the disk may make it susceptible to fragmentation (e.g., Rafikov 2005).

Spiral arms, particularly grand-design two-armed ones, are being revealed as common (up to $\sim 20 \%$ ) in high-resolution imaging surveys in both NIR scattered light (Dong et al. 2018b) and mm continuum observations (Andrews et al. 2018; Cieza et al. 2019; Huang et al. 2018; Kurtovic et al. 2018). It is therefore of critical importance to determine the physical mechanism, or possible mechanisms, that are driving them. Unfortunately, the two most widely considered scenarios, companion and GI, are both difficult to verify in individual systems. To confirm the former, direct imaging observations searching for companions are needed. Such observations are challenging (e.g., Testi et al. 2015; Maire et al. 2017), particularly if planets form in the "cold start" instead of the commonly assumed "hot start" scenario (Spiegel \& Burrows 2012). Therefore, except in rare cases (e.g., HD 100453, Dong et al. 2016; Wagner et al. 2018), arm-driving companions have not been confirmed.

To verify GI as the arm-driving mechanism, accurate measurements of the total mass of the gas disk are required. The most common avenue of estimating disk masses from sub$\mathrm{mm}$ dust continuum relies on knowledge of the dust-to-gas mass ratio and the optical properties of dust grains (Beckwith et al. 1990), both of which are highly uncertain, and can lead to underestimating the disk masses by a factor of up to $\sim 100$ (Forgan \& Rice 2013a). Estimating disk masses through ${ }^{13} \mathrm{CO}$ and $\mathrm{C}^{18} \mathrm{O}$ isotopolgues emission is possible (Williams \& Best 2014), but this method is model-dependent and suffers from uncertainties in, for example, the chemistry of CO (Ilee et al. 2011, 2017; Yu et al. 2017).

Given the difficulties in determining the origin of observed spiral arms directly and in individual systems, we explore the likelihood of observing GI-induced spiral arms in disks as a sample. We follow the time evolution of an isolated disk that has just emerged from its natal envelope to an age of $\sim 10 \mathrm{Myr}$. The system undergoes angular momentum transport primarily due to the GI. Our goal is to take a holistic approach. Rather than attempting to explain the morphologies of individual systems, we ask a broader question. Given its observability, and the observed occurrence rate of spirals in disks, how likely is it that GI is the dominant spiral-driving mechanism in protoplanetary disks?

\section{Method}

We begin with a one-dimensional model of an evolving selfgravitating disk and use this to obtain disk masses at times that are representative of evolutionary stages in the paradigm of evolving protoplanetary disks. We use this model to set the disk-to-star mass ratios of global, three-dimensional (3D) hydrodynamics simulations of self-gravitating disks at representative epochs. Once evolved for a few orbital periods at the disk outer edge, we perform radiative transfer calculations and generate synthetic images to predict how such systems would be observed by ALMA.

The disk is modeled in isolation. We define time as $t_{\text {tot }}=$ $t_{0}+t$, where $t_{\text {tot }}$ is the total time (i.e., system age), $t_{0}$ is the point at which disk accretion dominates over infall, and $t$ refers to simulation time (throughout the paper, "time" refers to $t$ ). Most likely, $t_{0}$ occurs during the late stages of Class 0 or early Class I phase, while a partial envelope may still be present. Prior to $t_{0}$, we can crudely think of the envelope as supplying mass at a constant rate to the disk, such that the disk maintains a time-independent surface density profile and therefore a constant total mass. After $t_{0}$, envelope infall has effectively ceased, disk accretion continues, draining the disk onto the central protostar.

\subsection{Time-dependent One-dimensional Model}

We use the one-dimensional model of Rice \& Armitage (2009), which evolves a self-gravitating protoplanetary disk under the assumption that the gravitational potential is fixed, angular momentum transport is primarily due to disk selfgravity, and the disk is in thermal equilibrium. The model does, however, assume that there is a minimum viscous $\alpha$, which could be produced via, e.g., the magnetorotational instability. It also includes a disk wind that dominates, and dissipates the disk mass, when the mass accretion rate is low. Full details are given in Rice \& Armitage (2009). However, we outline the basics of the model here.

Since we assume that the disk evolves pseudo-viscously, the surface density, $\Sigma(r, t)$, evolves according to (Lynden-Bell \& Pringle 1974; Pringle 1981)

$$
\frac{\partial \Sigma}{\partial t}=\frac{3}{r} \frac{\partial}{\partial r}\left[r^{\frac{1}{2}} \frac{\partial}{\partial r}\left(\nu \Sigma r^{\frac{1}{2}}\right)\right]
$$

where $\nu$ is the kinematic viscosity. We can express this viscosity as $\nu=\alpha c_{s} H$, where $\alpha \ll 1$ is the viscosity parameter (Shakura \& Sunyaev 1973). If the disk can maintain a quasi-steady state, 
Equation (3) can be integrated to give the steady-state mass accretion rate (Pringle 1981)

$$
\dot{M}=3 \pi \nu \Sigma \text {. }
$$

Viscosity generates dissipation in the disk at the rate (Bell \& Lin 1994)

$$
D(R)=\frac{9}{4} \nu \Sigma \Omega^{2}
$$

where $D(R)$ is per unit area per unit time. Assuming a quasisteady state, heating is balanced by cooling, with the cooling time, $t_{\text {cool }}$, given by (Gammie 2001)

$$
t_{\mathrm{cool}}=\frac{4}{9 \gamma(\gamma-1) \alpha \Omega},
$$

where $\gamma$ is the ratio of specific heats. The cooling time, $t_{\text {cool }}$, can also be expressed as $t_{\text {cool }}=U / \Lambda$, where $U$ is the internal energy per unit area,

$$
U=\frac{c_{s}^{2} \Sigma}{\gamma(\gamma-1)},
$$

and the cooling rate $\Lambda$ is given by (Pringle 1981; Hubeny 1990; Johnson \& Gammie 2003; Rice \& Armitage 2009)

$$
\Lambda=\frac{16 \sigma}{3}\left(T_{\text {mid }}^{4}-T_{0}^{4}\right) \frac{\tau}{1+\tau^{2}} .
$$

Here, $T_{0}=3 \mathrm{~K}$ and is assumed to come from a background irradiation source that prevents the midplane of the disk from cooling below this value (Stamatellos et al. 2007). The optical depth is approximated using

$$
\tau=\int_{0}^{\infty} d z \kappa\left(\rho_{z}, T_{z}\right) \rho_{z} \approx H \kappa(\bar{\rho}, \bar{T}) \bar{\rho},
$$

where $\kappa$ is the Rosseland mean opacity (obtained from Bell \& Lin 1994), $\bar{\rho}=\Sigma /(2 H)$, and $\bar{T}=T_{\text {mid }}$.

Closing Equations (3)-(9) is our assumption that the disk will settle into a self-gravitating state with $Q=1.5$. Given the surface density, we are therefore able to estimate the sound speed, the cooling timescale, the equilibrium heating rate and, hence, $\alpha$. In our model, we do not include that some of the mass flowing through the disk will accrete onto the central star, and increase its mass. We instead assume that mass accreted through the disk is completely lost in a jet. This does mean that our model is a simplification, however, most of the mass is accreted within the first $10^{5}$ years (Rice et al. 2010), so this will not significantly change the relationship between system age and strength of the GI. We are, essentially, considering the best case scenario, where $q$ remains as high as possible for as long as possible.

\subsection{Smoothed Particle Hydrodynamics (SPH) Simulations and Emission Maps}

The SPH simulations (Gingold \& Monaghan 1977; Lucy 1977) are based on the code developed by Bate et al. (1995), updated to include a hybrid radiative transfer method that approximates realistic heating and cooling (Forgan et al. 2009; Stamatellos \& Whitworth 2009). Essentially, the polytropic cooling approximation of Stamatellos et al. (2007) is combined with the flux-limited diffusion method of Mayer et al. (2007), which together can account for the local optical depth of the system as well as the energy exchange between particles.

The disk is heated through $P d V$ work, and we assume the central star mass is $1 M_{\odot}$. We run seven models, with disk-tostar mass ratios of $q=0.5,0.25,0.2,0.175,0.125,0.1$, and 0.075 .

Each disk has $5 \times 10^{5}$ particles, initially located between 6 and $60 \mathrm{au}$, and the initial surface density profile and initial sound speed profile are $\Sigma \propto r^{-1}$ and $c_{s} \propto r^{-1 / 4}$.

We use the TORUS radiation transport code (Harries et al. 2004; Kurosawa et al. 2004; Haworth et al. 2015) to calculate continuum emission maps of the SPH disks using the dust temperatures directly from the SPH simulations. To do so, a 3D grid must be constructed from the particle distribution. Full details of this are given in Rundle et al. (2010), but the basic idea is to begin with one cell centered on the entire disk, and then to repeatedly divide this cell according to a resolution criterion (for example, resolve $n$ particles per cell). The original cell is divided once in each dimension, resulting in $2^{D}$ child cells, where $D$ is the number of dimensions. We resolve the mass represented by every active particle on the grid, resulting in $\sim 500,000$ grid cells.

The dust in our model is Draine \& Lee (1984) silicates, with a grain size distribution given by

$$
n(a) \propto a^{-q} \quad \text { for } \quad a_{\min }<a<a_{\max },
$$

where $a_{\min }$ and $a_{\max }$ are the minimum and maximum grain sizes, taken to be $0.1 \mu \mathrm{m}$ and $2000 \mu \mathrm{m}$, respectively, and $q=3.5$, the standard power-law exponent for the ISM (Mathis et al. 1977). We assume a dust-to-gas ratio of 1:100 everywhere in the disk (Meru et al. 2017; Tomida et al. 2017). Previous numerical work has shown that it is possible to increase the fraction of grains present in the spiral arm of a selfgravitating disk through particle trapping (Rice et al. 2004). Regardless, we do not expect this effect to significantly affect $\sim$ mm grains. We do, however, discuss the implications of our assumptions in the summary and discussion section.

\subsection{Detecting Substructure in Synthetic ALMA Images}

The emission maps generated by TORUS are used as inputs to the ALMA simulator included in CASA (ver 4.7.2; McMullin et al. 2007), and all disks were imaged at a distance of $139 \mathrm{pc}$, as if in the $\rho$-Ophiuchus region (Mamajek 2008). We synthesize observations centered on $230 \mathrm{GHz}$ (band 6), chosen such that there is a balance between the disk being more optically thin (where longer wavelengths are preferred), and obtaining a higher signal-to-noise ratio (where shorter wavelengths are preferred). We choose the maximum bandwidth available in ALMA band $6(7.5 \mathrm{GHz})$, since this maximizes sensitivity. We corrupt the visibilities with thermal noise by using the Atmospheric Transmission at Microwaves (ATM) code (Pardo et al. 2001). The total integration time, beam sizes, and precipitable water vapour (PWV) values are given in Table 1.

For the $q=0.5$ and $q=0.25$ disks, PWV values are chosen as an estimate from the ALMA sensitivity calculator at the fifth octile for this wavelength. We assume exceptional observing conditions in the case of $q=0.175$ and $q=0.125$, motivated by $\mathrm{PWV}<0.7 \mathrm{~mm} 50 \%$ of the time in August. We use the simobserve and simanalyze routines in CASA, which perform a standard clean, using Briggs weighting of the 
Table 1

Parameters Used to Generate Synthetic Observations

\begin{tabular}{llccl}
\hline \hline$t$ (yr) & $q$ & $\begin{array}{c}\text { int. } \\
\text { time }(\mathrm{hr})\end{array}$ & $\begin{array}{c}\text { Beam } \\
\text { Size }\end{array}$ & $\begin{array}{l}\text { PWV } \\
(\mathrm{mm})\end{array}$ \\
\hline$\sim 10^{3}$ & 0.5 & 1 & $0.1^{\prime \prime} \times 0.09^{\prime \prime}$ & 1.796 \\
$\sim 10^{4}$ & 0.25 & 12 & $0.12^{\prime \prime} \times 0.08^{\prime \prime}$ & 1.796 \\
$\sim 10^{5}$ & 0.175 & 72 & $0.05^{\prime \prime} \times 0.04^{\prime \prime}$ & 0.45 \\
$\sim 10^{6}$ & 0.125 & 120 & $0.03^{\prime \prime} \times 0.02^{\prime \prime}$ & 0.45 \\
\hline
\end{tabular}

Note. From left to right is simulation time in years, disk-to-star mass ratio, total integration time on source in hours, beam size in arcseconds, and precipitable water vapour in $\mathrm{mm}$.

visibilities with a robust parameter of zero and multi-scale deconvolution (Rau \& Cornwell 2011).

We generate synthetic images at four stages of the disk lifetime, when $q=0.5\left(t \sim 10^{3} \mathrm{yr}\right), q=0.25\left(t \sim 10^{4} \mathrm{yr}\right)$, $q=0.175\left(t \sim 10^{5} \mathrm{yr}\right)$, and $q=0.125\left(t \sim 10^{6} \mathrm{yr}\right)$. We begin with a shorter integration time on the most massive disk (1 hr), since the most massive disk has the largest total flux and so smaller integration times will suffice. As the disk mass decreases, we use progressively longer integration times. Essentially, the observing parameters were varied in order to maximize the detection of spiral arms in the shortest integration time, so the images presented here show the most clear results with optimal use of resources.

In all cases, the surface density profile of a self-gravitating disk in quasi-steady equilibrium is steep, which makes it difficult to observe the fainter non-axisymmetric structure away from the center of the disk. To reduce the overall range in the image (which enhances the fainter features), we convolve each image with a two-dimensional (2D) Gaussian, and then subtract this from the original image to obtain the "residuals" (i.e., the unsharped image masking operation; Malin 1977; see applications in, e.g., Pérez et al. 2016).

\section{Results}

\subsection{Temporal Behavior on Secular Timescales}

We ran seven global SPH simulations, with initial disk masses taken from seven points in time from Figure 1. Doing so allowed us to capture the secular behavior of the system, while simulating for several outer orbits allowed us to capture behavior occurring on dynamical timescales. While the onedimensional (1D) models of Rice \& Armitage (2009) provide surface density profiles of the quasi-steady-state disks (expected roughly after the thermal timescale of the system), we did not use them for the initial conditions. This is because the azimuthally averaged surface density profiles of the 3D simulations are slightly different due to capturing the nonaxisymmetric structure of the system (i.e., large-scale, global spiral arms). In both the 1D and the 3D case, the qualitative behavior is the same. Beginning with some imposed surface density profile, the system evolves to a surface density profile in the quasi-steady state that is independent of the initial configuration of the system.

We begin with the disks in a $\Sigma \propto r^{-1}$ configuration and allow them to evolve to their quasi-steady profile. The temperature profile is initially $T \propto r^{-1 / 2}$, with the temperature normalized such that the minimum value of $Q$ is $Q_{\min }=2$. This is a local parameter, so $Q=Q_{\min }$ only at the disk outer edge. The disks subsequently cool until $Q$ is low enough for GI to set in, which

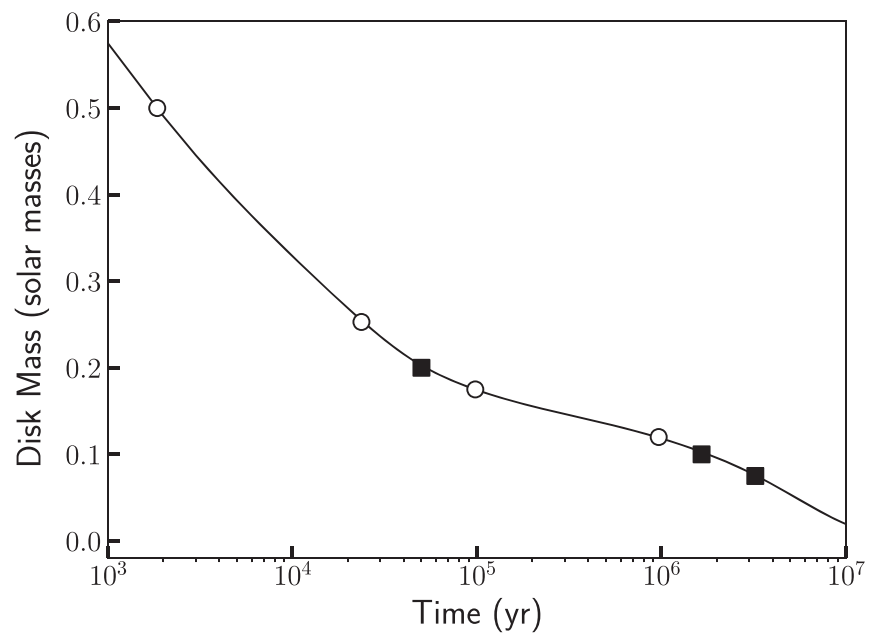

Figure 1. The time-dependent mass evolution of a self-gravitating disk orbiting a $1 M_{\odot}$ star, obtained self-consistently using the models of Rice \& Armitage (2009). The line shows the disk mass as a function of time, with markers showing the points that were used to set the disk masses of the SPH simulations. Circular markers show points in time used for $\mathrm{SPH}$ simulations and synthetic observations. Square markers show points in time used only for SPH simulations.

then provides heating. The heating and cooling ultimately roughly balance and the disk settles into a quasi-steady state.

The top row of Figure 2 shows the surface density structure for four simulations with different total disk masses. The second row of Figure 2 shows the fractional physical amplitude of the spiral between $R=40$ au and $R=45 \mathrm{au}$,

$$
\frac{\delta \Sigma}{\Sigma}=\frac{\delta \Sigma(\phi)_{r=40-45 \mathrm{au}}}{\Sigma_{r=40-45 \mathrm{au}}}=\frac{\sum(\phi)_{r=40-45 \mathrm{au}}-\sum_{r=40-45 \mathrm{au}}}{\sum_{r=40-45} \mathrm{au}}
$$

where $\Sigma_{r=40-45 \text { au }}$ is the azimuthally averaged surface density in a ring between $R=40$ au and $R=45$ au. It is clearly shown that as the disk "evolves" in time: both the number of peaks increases and the physical amplitude of the spiral decreases.

The third row shows the synthetic ALMA residuals (Section 2.3), which display the non-axisymmetric structure in the disk that would be observed in a real ALMA observation. Figure 3 shows the synthetic images without the unsharp mask technique applied, showing the necessity of applying the unsharp mask in order to enhance the fainter features and reduce the overall range of the image. The residuals clearly show that as the number of spiral arms increases, and their physical amplitude decreases, it becomes increasingly difficult to detect non-axisymmetric structure, due to a decrease in overall flux, a decrease in contrast between the arm and interarm region, and the smaller physical scale of the spirals.

When the disk mass is large $(q=0.5)$, and the spirals are loosely wound, an hour on source is sufficient to detect the substructure, suggesting that future protoplanetary disk surveys that perform hour-long integrations should be able to detect substructure due to GI.

As the disk mass decreases, the spirals become increasingly faint and more tightly wound, requiring both a smaller beam size and a far longer integration time. When $q=0.25,12 \mathrm{hr}$ on source is required. When disk mass is decreased further $(q=0.175), 72 \mathrm{hr}$ is required on source to detect substructure, with a more compact beam $\left(0{ }^{\prime \prime} 05 \times 0\right.$. " 04$)$, as well as a smaller amount of PWV in the atmosphere. Observing such structure would require a dedicated integration on a deliberately targeted 

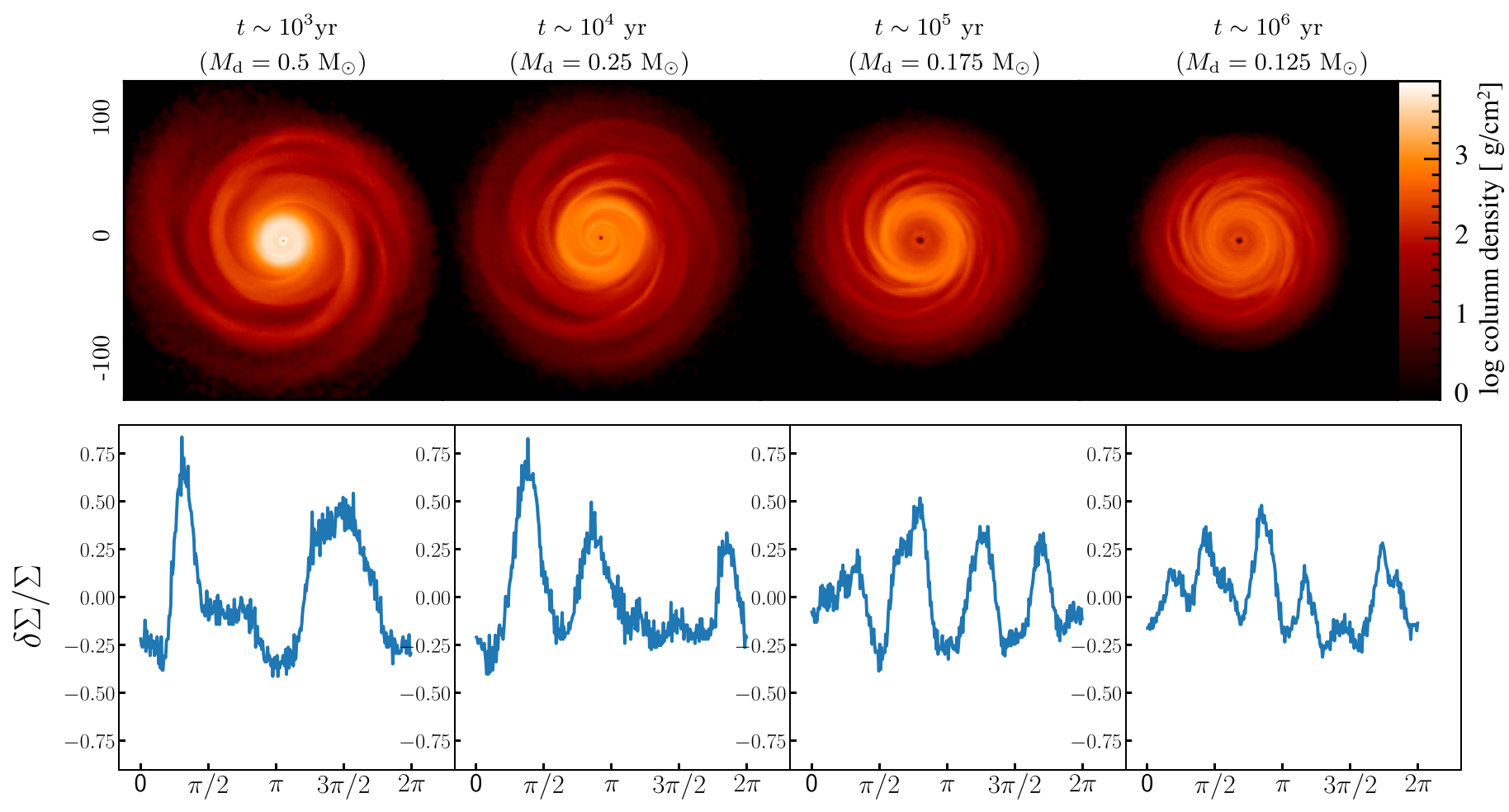

1 hour

12 hours

72 hours

120 hours
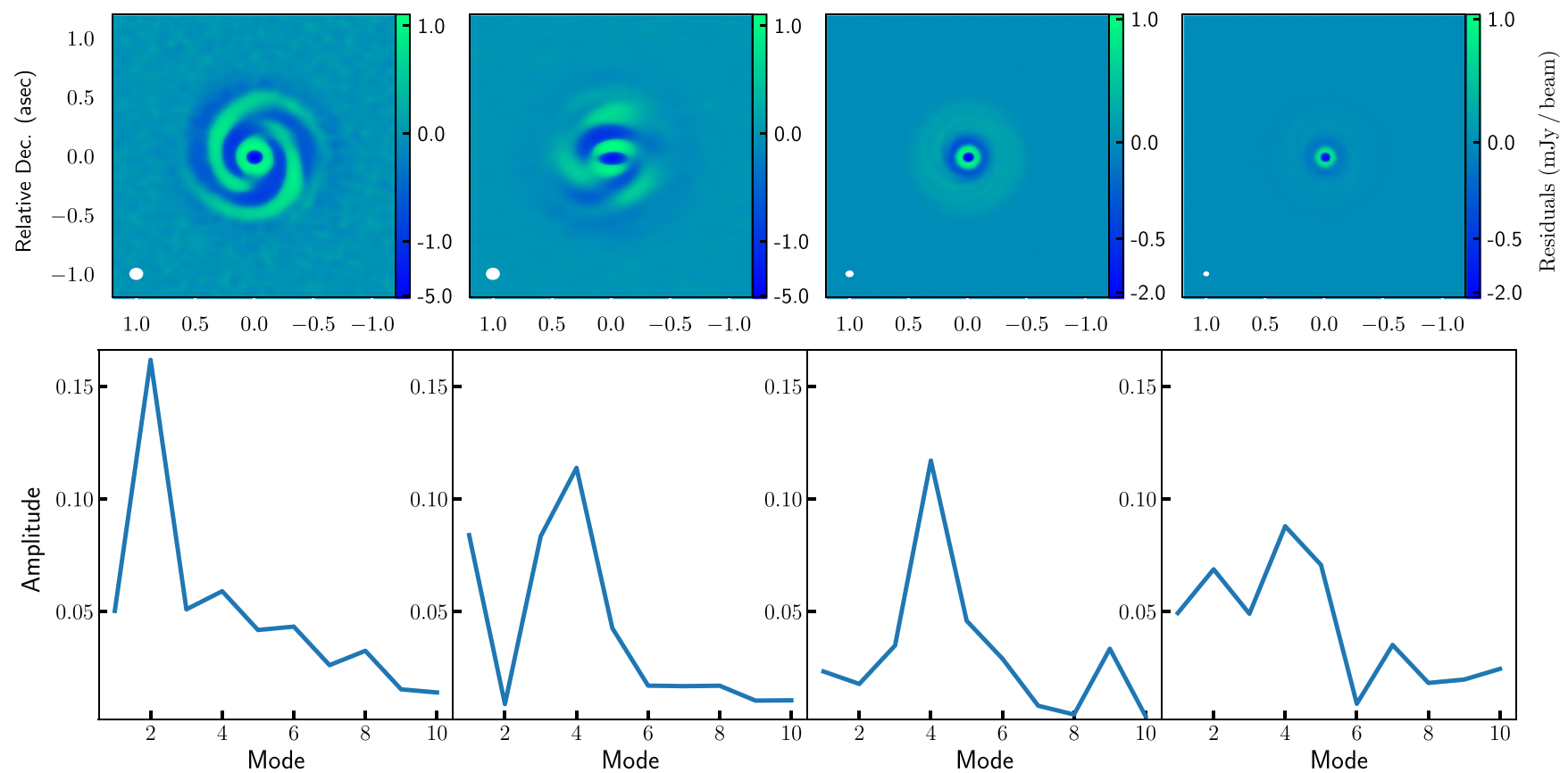

Figure 2. Top row: surface density structure of the disks considered. Second row: Azimuthal surface density variation between $R=40$ au and $R=45$ au (Equation (11)). Third row: residuals of synthetic ALMA observations that have been convolved with a 2D Gaussian and then subtracted from the original (Section 2.3). This is to enhance the fainter substructure in the outer parts of the disk. Non-axisymmetric structure becomes increasingly difficult to detect as (1) spiral amplitudes decrease (2) dominant $m$-mode increases. Bottom row: Fourier amplitude of each disk computed in a ring between $R=40$ au and $R=45$ au. Nonaxisymmetric structure is only visible in the ALMA residuals when there is sufficient power in the low $m$-modes.

source, which makes the detection of GI in protoplanetary disks with disk-to-star mass ratios of less than 0.25 unlikely. Finally, for the least massive disk $(q=0.125)$, no detection of substructure is visible even with an integration time of $120 \mathrm{hr}$.

This can also be understood in terms of the Fourier amplitude, which we calculate for each mode in a ring between $R=40$ au and $R=45$ au. This is representative of the majority of the disk, and a relatively thin ring is required to avoid the structure being averaged out. The results do not change if the location of the ring is varied. The Fourier amplitude, $A_{m}$, of each mode, $m$, is given by

$$
A_{m}=\left|\sum_{i=1}^{N_{\text {ring }}} \frac{e^{-i m \phi_{i}}}{N_{\text {ring }}}\right|,
$$



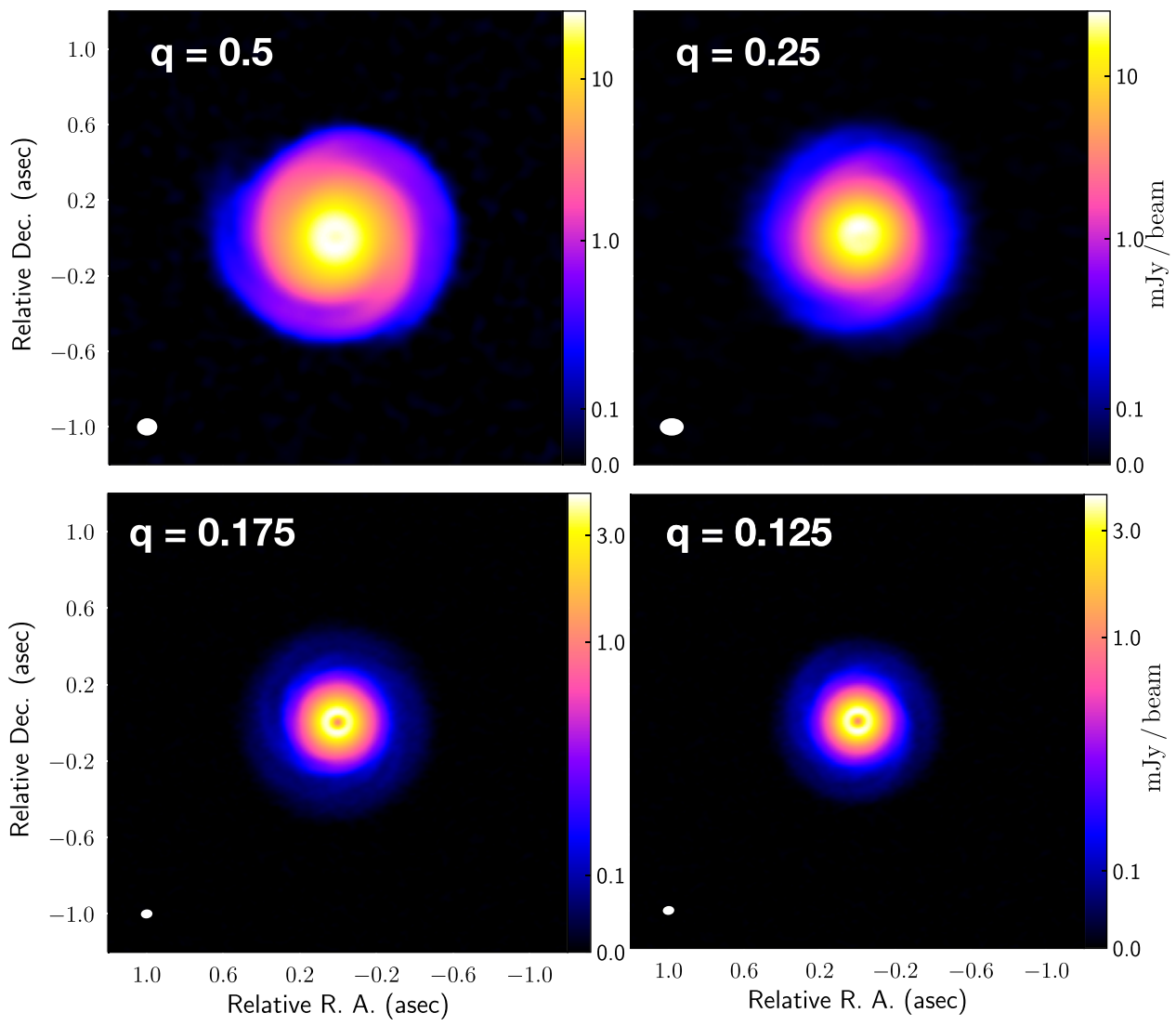

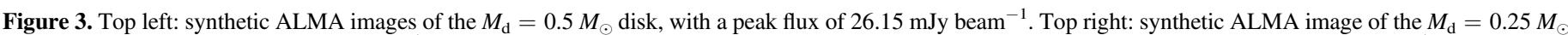

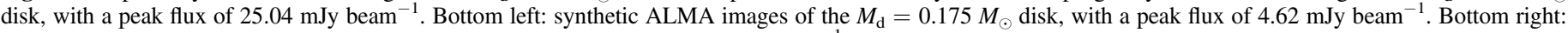
synthetic ALMA images of the $M_{\mathrm{d}}=0.125 M_{\odot}$, with a peak flux of $4.33 \mathrm{mJy}$ beam $^{-1}$.

where $N_{\text {ring }}$ is the number of particles in each ring and $\phi_{i}$ is the azimuthal angle of the $i$ th particle. The Fourier amplitude of the first 10 modes are displayed in the bottom row of Figure 2.

We can see that when the disk just emerges from its natal envelope and is at its most massive state (leftmost column), two grand-design global spirals are clearly visible, with most of the power in the $m=2$ Fourier mode. This is shown in the bottom left panel of Figure 2.

As the disk evolves, the amplitude of this mode decreases, and more power may be found in lower $m$-modes. For the $q=0.25 \mathrm{disk}$, this results in some non-axisymmetric structure remaining visible in the residual image, but not as clearly as when the $m=2$ mode dominates. As the disk continues to evolve, less and less power is in the lower $m$-modes, resulting in increased difficulty of detection.

In order to numerically quantify these results, we perform this Fourier analysis on seven simulations, each representing the disk at a time taken from Figure 1. We take the Fourier amplitude of each mode, and average it over $\sim 4$ orbital periods at the disk outer edge. The results are shown in Figure 4. We can see that up until a few $\times 10^{4}$ years $(\sim 100$ orbits at $R=100$ au), the $m=2$ mode dominates the spectrum. From the two leftmost columns of Figure 2, we can see that GI-induced structure is detectable when (1) the low $m$-modes $(m=2,3$, or 4) dominate the power spectrum, and (2) the Fourier Amplitude in these modes (i.e., surface density contrast in structures) is $\sim 0.1$ or larger.

In total, GI-induced structures remain readily detectable ( $\sim$ hours with ALMA) for the first few $10^{4}$ years after the

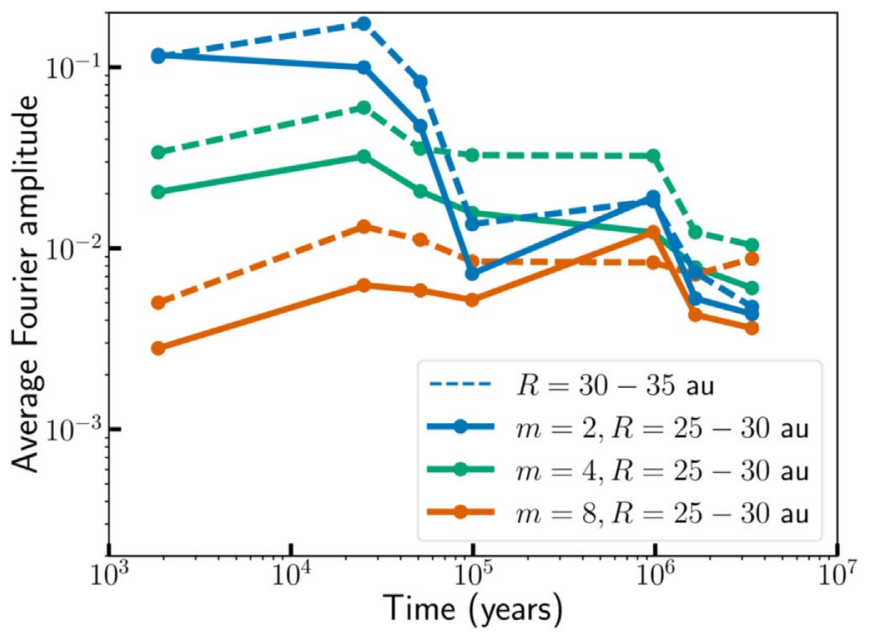

Figure 4. Fourier amplitudes of even modes as a function of time. As time increases, the amount of power in the lower $m$-modes decreases, which makes detecting this spiral structure with an instrument such as ALMA increasingly difficult. As long as the amplitude remains above $\sim 0.1$ for a low $m$-mode (where $m \leqslant 4$ ), GI spirals will be detectable by ALMA.

system has emerged from its nascent envelope, when $q$ stays above $\sim 0.25$. After this time $\left(\gtrsim 10^{5}\right.$ years $)$, detecting the substructure requires an integration time of $\sim$ days. Ultimately, the size scale of the substructure will drop below the resolution limit of ALMA, so even with increased integration time, resolving the substructure will not be possible. 


$$
\begin{aligned}
& t=10^{3} \text { years } \\
& M_{\mathrm{d}}=0.5 \mathrm{M}_{\odot}
\end{aligned}
$$

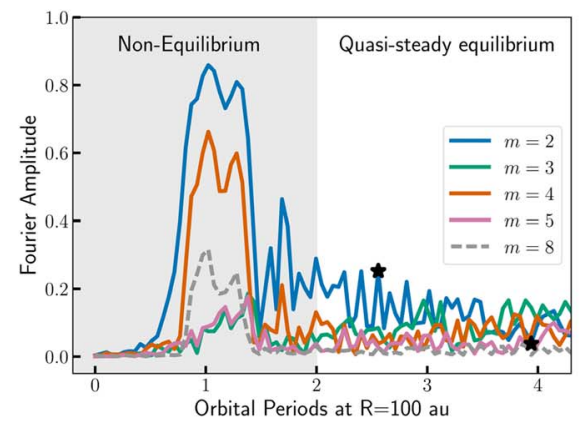

$t=10^{4}$ years

$M_{\mathrm{d}}=0.25 \mathrm{M}_{\odot}$

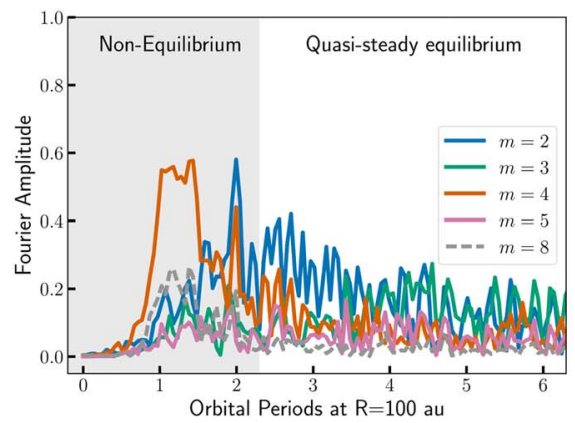

$t=4 \times 10^{6}$ years

$M_{\mathrm{d}}=0.075 \mathrm{M}_{\odot}$

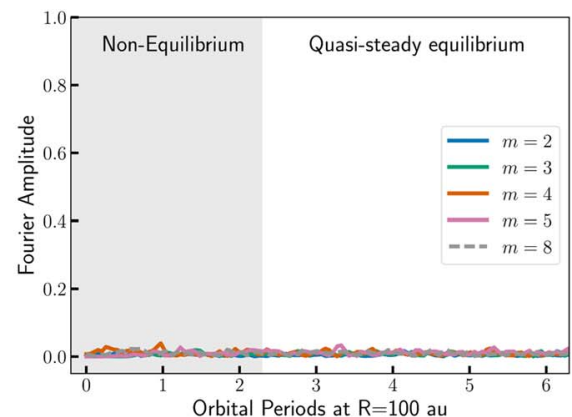

Figure 5. Fourier amplitudes of $m=2, m=4$, and $m=8$ modes for disks at $10^{3}$ years, $10^{4}$ years, and $10^{6}$ years. Each plot should be considered separately. For example, at $t=0$, the disk has undergone some evolution and infall from its nascent cloud, which increases the surface density such that large, transient spiral waves are produced until the system settles into a state of quasi-steady equilibrium. At later stages of the disk lifetime, there is very little power in the lower $m$-modes, which are more readily detectable by ALMA due to density contrast and larger spatial separation.

$$
\mathrm{A}_{(m=2)}=0.25 \quad \mathrm{~A}_{(m=2)}=0.03
$$
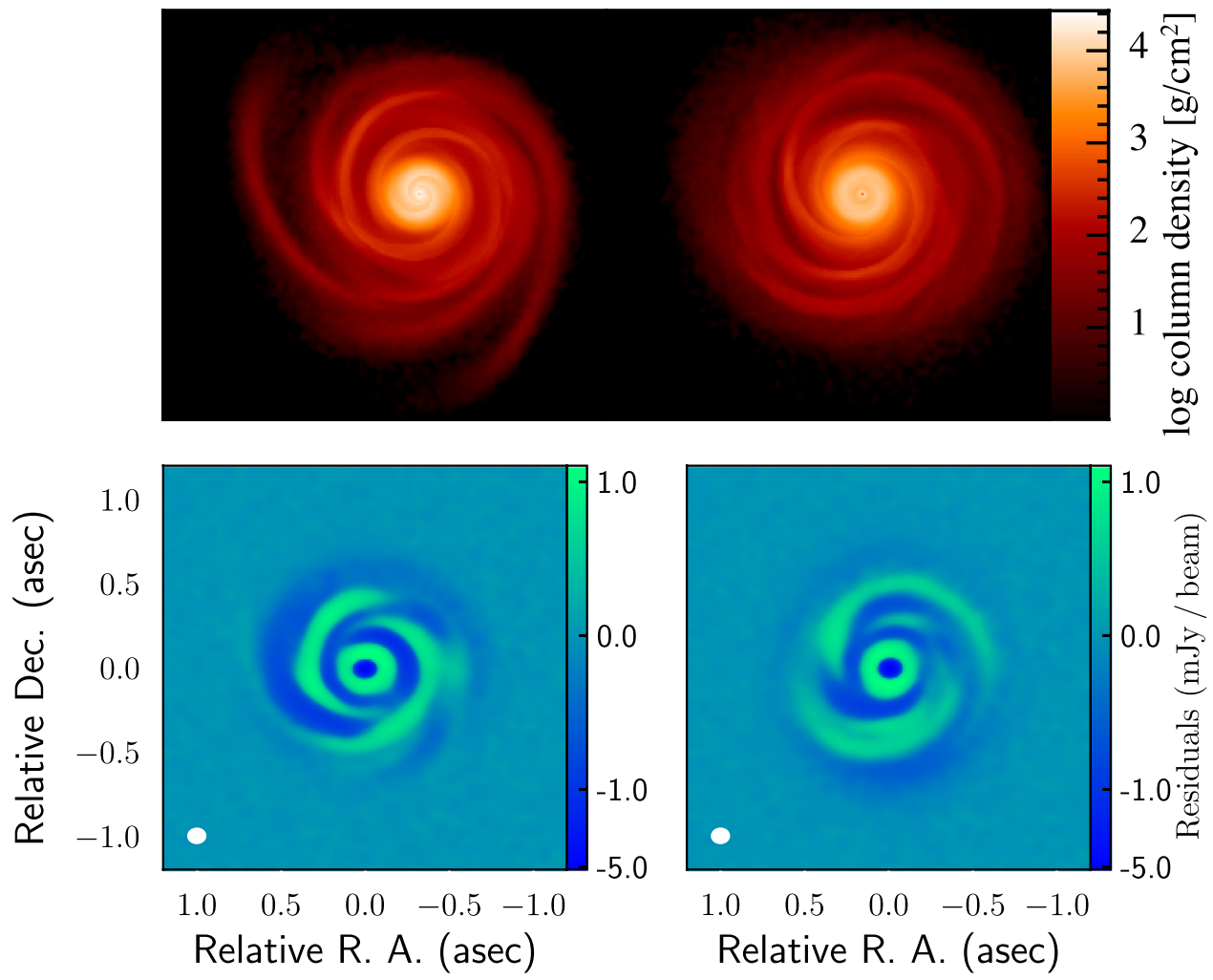

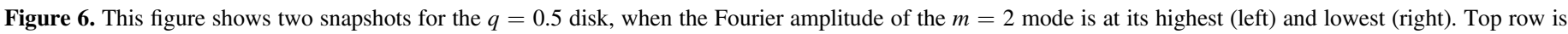

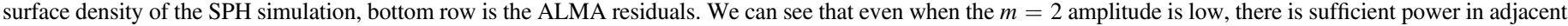
modes such that non-axisymmetric structure is still detectable.

\subsection{Temporal Behavior on Dynamical Timescales}

We now turn to the behavior of these systems on dynamical timescales. In Section 3.1, we assert that detection of spiral structure is easiest when the $m=2$ mode dominates the spectrum, so we examine if having a low amplitude in the $m=2$ mode will prevent detection, or alternatively, how else the power may be distributed in the mode spectrum so that detection is still possible.

Figure 5 shows the amplitude of the $m=2-5$ and 8 Fourier modes as a function of time for the $q=0.5,0.25$, and 0.075 disks. At $t=0$, all particles in the disk are in exact Keplerian rotation. The more massive disks then undergo a period of violent relaxation where large, global $m=2$ modes rapidly redistribute angular momentum until the disk settles into a quasi-steady state. We select two points in time for the $q=0.5$ disk, shown in the leftmost panel of Figure 5, marked with black stars. The first point, at $\sim 2.5$ outer orbital periods, is when the $m=2$ Fourier amplitude is highest. The second point, at $\sim 4$ outer orbital periods, is when the $m=2$ Fourier amplitude is lowest during the quasi-steady state. We note, however, that in the $q=0.5$ case, even as power decreases in the $m=2$ mode, power can still be found in the $m=3-4$ modes. 
We generate synthetic "residual" images at these two epochs in Figure 6. The top row shows the surface density structure of the disks, and the bottom row shows the ALMA residuals. In both cases, spiral structure is clearly detectable, despite the order of magnitude difference in the amplitude of the $m=2$ mode. We therefore conclude that the detectability of GI spirals has little dependence upon behavior exhibited on the dynamical timescale of the system. As long as the disk mass is high enough, there will be sufficient power in the low $m$-modes and the spirals will remain detectable, even if the amplitude oscillates between modes.

\subsection{Caveats}

Interpreting disk observations is impossible without the use of numerical models, and generating these models is challenging. To properly model a system, we require full polychromatic radiation hydrodynamics, chemistry, as well as dust dynamics and back reaction. To perform all of these is not, at the time of writing, computationally possible (Haworth et al. 2016). Although we use a state-of-the-art radiative transfer approximation, we do not consider chemistry, and we assume that the dust and gas are well mixed.

In a self-gravitating disk, spiral arms are able to trap grains of certain sizes. This trapping is most effective for grain sizes of $\sim 10-100 \mathrm{~cm}$ in a typical self-gravitating disk (Rice et al. 2004), which contribute orders of magnitude less emission at $\sim$ mm wavelengths due to their low mm opacity (Draine 2006) when compared to $\mathrm{mm}$ grains. Therefore, since trapping in the regime of interest for our parameters is expected to be small, we assume that the dust and gas are well mixed. Future simulations with gas plus mm-sized dust are needed to quantify the effect.

\section{Summary and Discussion}

We have performed a series of 3D global SPH simulations of a self-gravitating disk at different stages, with the disk masses taken from the 1D models of Rice \& Armitage (2009). Essentially, this is equivalent to taking snapshots in time of a disk undergoing secular evolution. The 1D models captured the long-term evolution, while the 3D simulations captured the detailed disk structure and dynamical, transient effects. We have performed synthetic ALMA observations on these simulations, and used the unsharped image masking method to highlight asymmetric structures.

Our main conclusion is that in isolated systems, where evolution is driven primarily by GI, high amplitude, symmetric two-armed spirals should be rare. Such arms only last for a few $\times 10^{4}$ years after envelope dispersal, due to the rapid evolution of such massive disks. In these same systems, lower amplitude, multi-arm spirals can persist for much longer ( $\sim$ Myr timescales). However, long integrations (i.e., up to $72 \mathrm{hr}$ on source) and high angular resolution (i.e., $\lesssim 0$ ". 05 ) is needed to detect them.

A fundamental limitation of our investigation is that these are gas-only simulations, and we assume gas and dust are perfectly coupled. In reality, as discussed in Section 3.3, there would be at least some dust enhancement due to trapping at local pressure maxima.

It is worth noting that as the disk mass decreases, the temperature at which $Q \sim 1$ will also decrease. Therefore, for lower disk masses (i.e., older disks), even a modest amount of external irradiation can wash out the spiral structure. In the environment where these objects are found, it is therefore increasingly difficult to maintain spiral structure with age.

Our further conclusions are as follows:

1. GI can produce detectable structure in the residuals of ALMA images taken with $\sim$ hour-long integrations when $q=M_{\text {disk }} / M_{*} \gtrsim 0.25$ and $R \lesssim 100$ au. Fourier analysis shows that this corresponds to a minimum Fourier amplitude (i.e., substructure surface density contrast) of $\sim 0.1$ in low $m$-modes with $m \lesssim 4$.

2. For an isolated system (i.e., insubstantial infall from envelope), the phase with readily detectable GI-induced structure lasts for a few $\times 10^{4}$ years after the infall has ceased (i.e., disk accretion rate surpasses infall rate).

3. After this phase, dedicated $\sim$ day-long integrations are able to detect substructure in disks with $q \sim 0.175$, corresponding to $10^{5}$ years after the cessation of infall. As disk mass continues to decrease, eventually substructure starts to exist on length scales below that of the highest ALMA resolution, so it cannot be detected.

4. Temporal variation of Fourier mode amplitudes on dynamical timescales does not affect whether ALMA is able to detect the spirals.

Among protoplanetary disks imaged in scattered light, $\sim 10 \%-20 \%$ have been found to host two-armed spirals (Dong et al. 2018b, and more with multi-armed spirals). In Section 1, we posed the question "how likely is it that GI is the dominant spiral-driving mechanism in protoplanetary disks, given its observability and the observed occurrence rate of spirals in disks?"

Our models show that for two-armed spirals observed in dust continuum emission to be caused by GI, the system must either still be embedded and receiving mass via infall so as to maintain a high disk-to-star mass ratio, or have emerged from its natal envelope within the last a few $\times \sim 10^{4}$ years, i.e., approximately $1 \%$ of the typical age of disks.

If the system is embedded in an envelope, then it is possible that infall from this envelope can drive power into the lower $m$ modes of the disk (Harsono et al. 2011), which would probably increase the ease with which these spirals would be detected.

Without diving into the specifics of each individual disk, we conclude that GI is unlikely to be the dominate mechanism driving observed spiral arms to date.

This work has shown that it is very difficult to detect spirals in GI disks in the continuum with ALMA, when the disk-tostar mass ratio drops below $q \sim 0.125$, which would typically correspond to times $t \gtrsim 1$ Myr. Previous work has shown that some of the conditions that we demonstrate here would also be true for NIR scattered light. Specifically, that the disk be massive $(q \gtrsim 0.25)$, compact, and have a relatively high accretion rate $\left(\gtrsim 10^{-6} M_{\odot} \mathrm{yr}^{-1}\right.$; Dong et al. 2015a) in order to drive low- $m$ spirals.

However, even a very small amount of remaining nascent envelope can obscure the disk at NIR wavelengths. To clearly image the surface of the disk requires that there is virtually no envelope present, which probably requires $q \lesssim 0.1$. If such disks are imaged in NIR scattered light, they should display a high number of spiral arms, since $m \sim 1 / q$. Therefore, it is unlikely that GI can explain objects such as MWC 758 (Benisty et al. 2015) or SAO 206462 (Garufi et al. 2013; Stolker et al. 2016), since these objects show prominent $\sim 2$-armed spirals. 
GI may, however, offer an explanation for systems that display multiple, weaker spirals arms in scattered light, such as AB Aur (Hashimoto et al. 2011). The measured disk mass of $\mathrm{AB}$ Aur from mm continuum observations is $\sim 20 M_{J}$ (Henning et al. 1998), placing the disk-to-star mass ratio at $q \sim 0.01$. This is about 5-10 times fewer than the lower limit necessary for GI to be active. However, disk masses inferred from $\mathrm{mm}$ dust observations have many uncertainties. If grain growth has occurred, which may be reasonably likely in a system that is $\sim$ Myr old, then the disk is expected to be optically thick out to $\sim 3 \mathrm{~mm}$ wavelengths. This can result in the underestimation of disk mass by an order of magnitude (Forgan \& Rice 2013a; Dunham et al. 2014; Evans et al. 2017; Galván-Madrid et al. 2018). If this is the case for a system such as AB Aur, then the system may be self-gravitating.

Recently, it has come to light that protoplanetary disks appear not to be massive enough to form the known exoplanet population (Manara et al. 2018). Either disks are being continually replenished from their environment (an unseen envelope), or cores of planets form very rapidly (between 0.1 and $1 \mathrm{Myr}$ ), and a large amount of gas is expelled shortly after their formation. If the ringed structure of systems such as HL Tau (ALMA Partnership et al. 2015) is due to planet formation (Dipierro et al. 2015b), then the latter is certainly possible, although no mechanism is known that would remove so much mass from the system on such a short timescale, which would only dominate after the formation of planetary cores. However, this problem is solved, and it does not challenge the current planet formation paradigm, if it is simply the case that disk masses are being systematically underestimated, as we have discussed above.

Although the work presented in this paper does not focus on fragmentation due to GI, it is worth noting that the work of Manara et al. (2018) finds two distinct populations for single and multiple exoplanetary systems, which, as they suggest, may point to a different formation mechanism for single exoplanets. Simply put, single exoplanets around low-mass stars can have masses that are comparable to their host star's mass, which is never observed in multi-planet systems. This seems to be consistent with the current understanding of planet formation through GI, that these objects are, essentially, failed companion stars (Kratter et al. 2010).

Previous numerical investigations have found that fragmentation occurs in GI disks that extend beyond $R \gtrsim 50-100$ au (Matzner \& Levin 2005; Rafikov 2005; Whitworth \& Stamatellos 2006; Clarke 2009; Clarke \& Lodato 2009; Kratter et al. 2010; Forgan \& Rice 2011; Hall et al. 2016, 2017, 2018).

Therefore, if a disk has spiral structure that extends beyond $\sim 100 \mathrm{au}$, it is unlikely to be caused by GI. Although irradiation beyond these radii reduces the local effective gravitational stress, and, therefore, the amplitude of the spiral arms (see, e.g., Hall et al. 2016), it does not prevent fragmentation (Rice et al. 2011). As irradiation increases, the disk behaves more like an isothermal system. As such, even large-amplitude spirals do little to dissipate thermal energy and redress the thermal balance (Kratter \& Murray-Clay 2011), resulting in nonlinear growth of the spirals and ultimately fragmentation.

The results presented here are consistent with the results of Hall et al. (2016), who found that detecting signatures of disk self-gravity with ALMA required the disk to exist in a very narrow region of parameter space, where the spiral wave amplitudes are large enough to produce detectable features, but not so large as to cause the disk to fragment. In this work, we have examined the region of parameter space where the semianalytical approach of Hall et al. (2016) would not have been valid, i.e., we have simulated global angular momentum transport by global (loosely wound, low $m$ ) spiral arms, rather than only considering the local regime (tightly wound spiral arms, high $m$ ) that can be described by a semi-analytical model. Essentially, in Hall et al. (2016), it was found that it is difficult to detect spiral arms caused by GI in the local regime $(q \lesssim 0.25)$, and we again find this result in this work.

Until recently, the disks around Elias 2-27 (Pérez et al. 2016) and MWC 758 (Dong et al. 2018a) were the only confirmed cases of $m=2$ spiral arms in protoplanetary disks imaged in mm continuum emission. However, the DSHARP (Disk Substructures at High Angular Resolution Project) ALMA survey (Andrews et al. 2018) has revealed four new instances of spiral arm structure in $1.25 \mathrm{~mm}$ emission in protoplanetary disks, around the systems IM Lup, WaOph 6, HT Lup A, and AS $205 \mathrm{~N}$, as well as a more high-resolution observation of Elias 2-27. HT Lup A and AS $205 \mathrm{~N}$ are multidisk systems, so it is likely that interactions between these components has given rise to the spiral structures present (Kurtovic et al. 2018).

The Elias 2-27, IM Lup, and WaOph 6 systems, however, have no known companions. Although it may be possible that the $m=2$ spiral structure present in these systems is due to GI, it has traditionally been thought that such Class II systems would be too low-mass to be susceptible to GI. This is compounded by the fact that measurements of the Toomre parameter for Elias 2-27 and IM Lup indicate that these disks should be stable to GI (Cleeves et al. 2016; Pérez et al. 2016). Furthermore, the recent high-resolution observations of these systems have revealed annular substructure in all three disks (Huang et al. 2018), in addition to the $m=2$ spiral arm pattern. It is difficult to explain the coexistence of spirals and annuli together with GI alone, suggesting that either GI is not acting, or it is present in conjunction with another mechanism responsible for the annular substructure.

Finally, the age estimates for Elias 2-27, Im Lup, and WaOph 6 are $0.8 \mathrm{Myr}$, $0.5 \mathrm{Myr}$, and $0.3 \mathrm{Myr}$, respectively (Luhman \& Rieke 1999; Eisner et al. 2005; Alcalá et al. 2017). The work we have presented here has shown that it is unlikely that $m=2$ spiral arms caused by GI persist at these ages. Systems with a higher number of weak GI-induced spiral arms can persist for far longer, $\sim 10^{6}$ years. However, these systems are far more difficult to detect with ALMA because the low contrast requires high sensitivity, and the high $m$-modes demand high angular resolution. It is yet to be seen whether the required observing conditions and integration times are realistic or not.

C.H. is a Winton Fellow, and this research has been supported by Winton Philanthropies. This research used the ALICE2 High Performance Computing Facility at the University of Leicester. This research also used the DiRAC DIaL (Data Intensive at Leicester) facility. We would like to thank Daniel Price for his publicly available SPH plotting code SPLASH (Price 2007), which we have made use of in this paper. This project has received funding from the European Research Council (ERC) under the European Union's Horizon 2020 research and innovation programme (grant agreement No. 
681601). T.J.H. acknowledges funding from Exeter's STFC Consolidated Grant (ST/M00127X/1).

\section{ORCID iDs}

Cassandra Hall (i) https://orcid.org/0000-0002-8138-0425

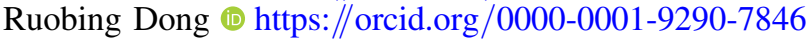
Tim J. Harries (i) https://orcid.org/0000-0001-8228-9503 Sean Brittain (1) https://orcid.org/0000-0001-5638-1330

\section{References}

Alcalá, J. M., Manara, C. F., Natta, A., et al. 2017, A\&A, 600, A20 ALMA Partnership, Brogan, C. L., Pérez, L. M., et al. 2015, ApJL, 808, L3 Andrews, S. M., Huang, J., Pérez, L. M., et al. 2018, ApJL, 869, L41 Andrews, S. M., Wilner, D. J., Espaillat, C., et al. 2011, ApJ, 732, 42 Andrews, S. M., Wilner, D. J., Hughes, A. M., Qi, C., \& Dullemond, C. P. 2009, ApJ, 700, 1502

Baruteau, C., Crida, A., Paardekooper, S.-J., et al. 2014, in Protostars and Planets VI, ed. H. Beuther et al. (Tucson, AZ: Univ. Arizona Press), 667

Bate, M. R., Bonnell, I. A., \& Price, N. M. 1995, MNRAS, 277, 362

Beckwith, S. V. W., Sargent, A. I., Chini, R. S., \& Guesten, R. 1990, AJ, 99, 924

Bell, K. R., \& Lin, D. N. C. 1994, ApJ, 427, 987

Benisty, M., Juhasz, A., Boccaletti, A., et al. 2015, A\&A, 578, L6

Cieza, L. A., Ruíz-Rodríguez, D., Hales, A., et al. 2019, MNRAS, 482, 698

Clarke, C. J. 2009, MNRAS, 396, 1066

Clarke, C. J., \& Lodato, G. 2009, MNRAS, 398, L6

Cleeves, L. I., Öberg, K. I., Wilner, D. J., et al. 2016, ApJ, 832, 110

Dipierro, G., Lodato, G., Testi, L., \& de Gregorio Monsalvo, I. 2014, MNRAS, 444, 1919

Dipierro, G., Pinilla, P., Lodato, G., \& Testi, L. 2015a, MNRAS, 451, 974

Dipierro, G., Price, D., Laibe, G., et al. 2015b, MNRAS, 453, L73

Dipierro, G., Ricci, L., Pérez, L., et al. 2018, MNRAS, 475, 5296

Dong, R., \& Fung, J. 2017, ApJ, 835, 146

Dong, R., Hall, C., Rice, K., \& Chiang, E. 2015a, ApJL, 812, L32

Dong, R., Liu, S.-y., Eisner, J., et al. 2018a, ApJ, 860, 124

Dong, R., Najita, J. R., \& Brittain, S. 2018b, ApJ, 862, 103

Dong, R., Zhu, Z., Fung, J., et al. 2016, ApJL, 816, L12

Dong, R., Zhu, Z., Rafikov, R. R., \& Stone, J. M. 2015b, ApJL, 809, L5

Dong, R., Zhu, Z., \& Whitney, B. 2015c, ApJ, 809, 93

Draine, B. T. 2006, ApJ, 636, 1114

Draine, B. T., \& Lee, H. M. 1984, ApJ, 285, 89

Dunham, M. M., Vorobyov, E. I., \& Arce, H. G. 2014, MNRAS, 444, 887

Durisen, R. H., Boss, A. P., Mayer, L., et al. 2007, in Protostars and Planets V, ed. B. Reipurth, D. Jewitt, \& K. Keil (Tucson, AZ: Univ. Arizona Press), 607

Eisner, J. A., Hillenbrand, L. A., White, R. J., Akeson, R. L., \& Sargent, A. I. 2005, ApJ, 623, 952

Evans, M. G., Ilee, J. D., Hartquist, T. W., et al. 2017, MNRAS, 470, 1828

Forgan, D., \& Rice, K. 2011, MNRAS, 417, 1928

Forgan, D., \& Rice, K. 2013a, MNRAS, 433, 1796

Forgan, D., \& Rice, K. 2013b, MNRAS, 432, 3168

Forgan, D., Rice, K., Stamatellos, D., \& Whitworth, A. 2009, MNRAS, 394, 882

Forgan, D. H., Hall, C., Meru, F., \& Rice, W. K. M. 2018a, MNRAS, 474, 5036 Forgan, D. H., Ilee, J. D., \& Meru, F. 2018b, ApJL, 860, L5

Galván-Madrid, R., Liu, H. B., Izquierdo, A. F., et al. 2018, ApJ, 868, 39

Gammie, C. F. 2001, ApJ, 553, 174

Garufi, A., Quanz, S. P., Avenhaus, H., et al. 2013, A\&A, 560, A105

Gingold, R. A., \& Monaghan, J. J. 1977, MNRAS, 181, 375

Gonzalez, J.-F., Laibe, G., Maddison, S. T., Pinte, C., \& Ménard, F. 2015 , MNRAS, 454, L36

Hall, C., Forgan, D., Rice, K., et al. 2016, MNRAS, 458, 306

Hall, C., Forgan, D., \& Rice, K. 2017, MNRAS, 470, 2517

Hall, C., Rice, K., Dipierro, G., et al. 2018, MNRAS, 477, 1004

Harries, T. J., Monnier, J. D., Symington, N. H., \& Kurosawa, R. 2004, MNRAS, 350, 565

Harsono, D., Alexander, R. D., \& Levin, Y. 2011, MNRAS, 413, 423
Hartmann, L., D'Alessio, P., Calvet, N., \& Muzerolle, J. 2006, ApJ, 648, 484 Hashimoto, J., Tamura, M., Muto, T., et al. 2011, ApJL, 729, L17

Haworth, T. J., Harries, T. J., Acreman, D. M., \& Bisbas, T. G. 2015, MNRAS, 453,2277

Haworth, T. J., Ilee, J. D., Forgan, D. H., et al. 2016, PASA, 33, e053

Henning, T., Burkert, A., Launhardt, R., Leinert, C., \& Stecklum, B. 1998, A\&A, 336, 565

Huang, J., Andrews, S. M., Pérez, L. M., et al. 2018, ApJL, 869, L43

Hubeny, I. 1990, ApJ, 351, 632

Humphries, R. J., \& Nayakshin, S. 2018, MNRAS, 477, 593

Ilee, J. D., Boley, A. C., Caselli, P., et al. 2011, MNRAS, 417, 2950

Ilee, J. D., Forgan, D. H., Evans, M. G., et al. 2017, MNRAS, 472, 189

Johnson, B. M., \& Gammie, C. F. 2003, ApJ, 597, 131

Kley, W., \& Nelson, R. P. 2012, ARA\&A, 50, 211

Kratter, K., \& Lodato, G. 2016, ARA\&A, 54, 271

Kratter, K. M., \& Murray-Clay, R. A. 2011, ApJ, 740, 1

Kratter, K. M., Murray-Clay, R. A., \& Youdin, A. N. 2010, ApJ, 710, 1375

Kurosawa, R., Harries, T. J., Bate, M. R., \& Symington, N. H. 2004, MNRAS, 351,1134

Kurtovic, N., Pérez, L., Benisty, M., et al. 2018, ApJL, 869, L44

Lin, D. N. C., \& Pringle, J. E. 1987, MNRAS, 225, 607

Lin, D. N. C., \& Pringle, J. E. 1990, ApJ, 358, 515

Lucy, L. B. 1977, AJ, 82, 1013

Luhman, K. L., \& Rieke, G. H. 1999, ApJ, 525, 440

Lynden-Bell, D., \& Pringle, J. E. 1974, MNRAS, 168, 603

Maire, A.-L., Stolker, T., Messina, S., et al. 2017, A\&A, 601, A134

Malin, D. F. 1977, AASPB, 16, 10

Mamajek, E. E. 2008, AN, 329, 10

Manara, C. F., Morbidelli, A., \& Guillot, T. 2018, A\&A, 618, L3

Mathis, J. S., Rumpl, W., \& Nordsieck, K. H. 1977, ApJ, 217, 425

Matzner, C. D., \& Levin, Y. 2005, ApJ, 628, 817

Mayer, L., Lufkin, G., Quinn, T., \& Wadsley, J. 2007, ApJL, 661, L77

McMullin, J. P., Waters, B., Schiebel, D., Young, W., \& Golap, K. 2007, in ASP Conf. Ser. 376, Astronomical Data Analysis Software and Systems XVI, ed. R. A. Shaw, F. Hill, \& D. J. Bell (San Francisco, CA: ASP), 127

Meru, F., Juhász, A., Ilee, J. D., et al. 2017, ApJL, 839, L24

Nayakshin, S. 2010, MNRAS, 408, L36

Okuzumi, S., Momose, M., Sirono, S.-i., Kobayashi, H., \& Tanaka, H. 2016, ApJ, 821, 82

Pardo, J. R., Cernicharo, J., \& Serabyn, E. 2001, ITAP, 49, 1683

Pérez, L. M., Carpenter, J. M., Andrews, S. M., et al. 2016, Sci, 353, 1519

Price, D. J. 2007, PASA, 24, 159

Pringle, J. E. 1981, ARA\&A, 19, 137

Rafikov, R. R. 2005, ApJL, 621, L69

Rau, U., \& Cornwell, T. J. 2011, A\&A, 532, A71

Rice, W. K. M., \& Armitage, P. J. 2009, MNRAS, 396, 2228

Rice, W. K. M., Armitage, P. J., Mamatsashvili, G. R., Lodato, G., \& Clarke, C. J. 2011, MNRAS, 418, 1356

Rice, W. K. M., Lodato, G., \& Armitage, P. J. 2005, MNRAS, 364, L56

Rice, W. K. M., Lodato, G., Pringle, J. E., Armitage, P. J., \& Bonnell, I. A. 2004, MNRAS, 355, 543

Rice, W. K. M., Mayo, J. H., \& Armitage, P. J. 2010, MNRAS, 402, 1740

Rundle, D., Harries, T. J., Acreman, D. M., \& Bate, M. R. 2010, MNRAS, 407, 986

Shakura, N. I., \& Sunyaev, R. A. 1973, A\&A, 24, 337

Spiegel, D. S., \& Burrows, A. 2012, ApJ, 745, 174

Stamatellos, D., \& Inutsuka, S.-i. 2018, MNRAS, 477, 3110

Stamatellos, D., \& Whitworth, A. P. 2009, MNRAS, 400, 1563

Stamatellos, D., Whitworth, A. P., Bisbas, T., \& Goodwin, S. 2007, A\&A, 475, 37

Stolker, T., Dominik, C., Avenhaus, H., et al. 2016, A\&A, 595, A113

Testi, L., Skemer, A., Henning, T., et al. 2015, ApJL, 812, L38

Tobin, J. J., Kratter, K. M., Persson, M. V., et al. 2016, Natur, 538, 483

Tomida, K., Machida, M. N., Hosokawa, T., Sakurai, Y., \& Lin, C. H. 2017, ApJL, 835, L11

Toomre, A. 1964, ApJ, 139, 1217

Wagner, K., Follete, K. B., Close, L. M., et al. 2018, ApJL, 863, L8

Whitworth, A. P., \& Stamatellos, D. 2006, A\&A, 458, 817

Williams, J. P., \& Best, W. M. J. 2014, ApJ, 788, 59

Yu, M., Evans, N. J., \& Dodson-Robinson, S. E. 2017, AAS Meeting Abstracts, 230, 402.05 